\title{
On the Chromatic Number of Matching Kneser Graphs
}

\author{
Alishahi, Meysam; Hossein, Hajiabolhassan
}

Published in:

Combinatorics Probability and Computing

Link to article, DOI:

10.1017/S0963548319000178

Publication date:

2019

Document Version

Early version, also known as pre-print

Link back to DTU Orbit

Citation (APA):

Alishahi, M., \& Hossein, H. (2019). On the Chromatic Number of Matching Kneser Graphs. Combinatorics Probability and Computing, 29(1), 1-21. https://doi.org/10.1017/S0963548319000178

\section{General rights}

Copyright and moral rights for the publications made accessible in the public portal are retained by the authors and/or other copyright owners and it is a condition of accessing publications that users recognise and abide by the legal requirements associated with these rights.

- Users may download and print one copy of any publication from the public portal for the purpose of private study or research.

- You may not further distribute the material or use it for any profit-making activity or commercial gain

- You may freely distribute the URL identifying the publication in the public portal

If you believe that this document breaches copyright please contact us providing details, and we will remove access to the work immediately and investigate your claim. 


\title{
On The Chromatic Number of Matching Graphs
}

\author{
Meysam Alishahi ${ }^{\dagger}$ and Hossein Hajiabolhassan* \\ $\dagger$ Department of Mathematics \\ University of Shahrood, Iran \\ meysam_alishahi@shahroodut.ac.ir \\ * Department of Mathematical Sciences \\ Shahid Beheshti University, G.C. \\ P.O. Box 19839-63113, Tehran, Iran \\ hhaji@sbu.ac.ir
}

\begin{abstract}
In an earlier paper, the present authors (2013) [1] introduced the altermatic number of graphs and used Tucker's Lemma, an equivalent combinatorial version of the Borsuk-Ulam Theorem, to show that the altermatic number is a lower bound for the chromatic number. A matching graph has the set of all matchings of a specified size of a graph as vertex set and two vertices are adjacent if the corresponding matchings are edge-disjoint. It is known that the Kneser graphs, the Schrijver graphs, and the permutation graphs can be represented by matching graphs. In this paper, as a generalization of the well-known result of Schrijver about the chromatic number of Schrijver graphs, we determine the chromatic number of a large family of matching graphs by specifying their altermatic number. In particular, we determine the chromatic number of these matching graphs in terms of the generalized Turán number of matchings.
\end{abstract}

Keywords: Chromatic Number, General Kneser Hypergraph,Turán Number. Subject classification: $05 \mathrm{C} 15(05 \mathrm{C} 65,55 \mathrm{U} 10)$

\section{Introduction}

For a hypergraph $\mathcal{H}$, the vertex set of the general Kneser graph $\mathrm{KG}(\mathcal{H})$ is the set of all hyperedges of $\mathcal{H}$ and two vertices are adjacent if the corresponding hyperedges are disjoint. It is known that any graph can be represented by various general Kneser graphs. In this paper, we show that one can determine the chromatic number of some graphs, by choosing some appropriate representations for them. In view of Tucker's Lemma, an equivalent combinatorial version of the Borsuk-Ulam Theorem, the present authors [1] introduced the altermatic number of Kneser hypergraphs. Moreover, they showed that it provides a tight lower bound for the chromatic number of Kneser hypergraphs. Next, they determined the chromatic number of some families of graphs, e.g., Kneser multigraphs, see [2].

A matching graph can be represented by the general Kneser graph $\operatorname{KG}(\mathcal{H})$, where the hyperedges of $\mathcal{H}$ are corresponding to all matchings of a specified size of a graph. It is worth noting that a Schrijver graph is a matching graph $\operatorname{KG}(\mathcal{H})$, where the hyperedge set of $\mathcal{H}$ is corresponding to all matchings of a specified size of a cycle. Hence, by determining the chromatic number of matching graphs, we generalize the well-known result of Schrijver about the chromatic number of Schrijver graphs.

\footnotetext{
${ }^{1}$ The research of Hossein Hajiabolhassan is supported by ERC advanced grant GRACOL.
} 
This paper is organized as follows. In Section 2, we set up notations and terminologies. In particular, we will be concerned with the definition of altermatic number and strong altermatic number and we mention some results about them. Also, we introduce the alternating Turán number of graphs which is a generalization of the generalized Turán number. Next, we introduce a lower bound for the chromatic number in terms of the alternating Turán number. In fact, if one can show that the alternating Turán number is the same as the generalized Turán number for a family of graphs, then one can determine the chromatic number of some family of graphs.

In Section 3, we study the chromatic number of matching graphs. In particular, as a generalization of the well-known result of Schrijver, we specify the chromatic number of a large family of matching graphs in terms of the generalized Turán number of matchings. Also, as a consequence, we determine the chromatic number of large permutation graphs.

\section{Notations and Terminologies}

In this section, we setup some notations and terminologies. Hereafter, the symbol $[n]$ stands for the set $\{1,2, \ldots, n\}$. A hypergraph $\mathcal{H}$ is an ordered pair $(V(\mathcal{H}), E(\mathcal{H}))$, where $V(\mathcal{H})$ is a set of elements called vertices and $E(\mathcal{H})$ is a set of nonempty subsets of $V(\mathcal{H})$ called hyperedges. Unless otherwise stated, we consider simple hypergraphs, i.e., $E(\mathcal{H})$ is a family of distinct nonempty subsets of $V(\mathcal{H})$. A vertex cover $T$ of $\mathcal{H}$ is a subset of its vertex set which meets any hyperedge of $\mathcal{H}$. Also, a $k$-coloring of a hypergraph $\mathcal{H}$ is a mapping $h: V(\mathcal{H}) \longrightarrow[k]$ such that for any hyperedge $e$, we have $|\{h(v): v \in e\}| \geq 2$, i.e., no hyperedge is monochromatic. The minimum $k$ such that there exists a coloring $h: V(\mathcal{H}) \longrightarrow[k]$ is called the chromatic number of $\mathcal{H}$ and is denoted by $\chi(\mathcal{H})$. Note that if the hypergraph $\mathcal{H}$ has some hyperedge with cardinality 1 , then there is no $k$-coloring for any $k$. Therefore, we define the chromatic number of such a hypergraph to be infinite. A hypergraph $\mathcal{H}$ is $k$-uniform, if all hyperedges of $\mathcal{H}$ have the same size $k$. By a graph $G$, we mean a 2 -uniform hypergraph. Also, let $o(G)$ denote the number of odd components of the graph $G$. For brevity, we use $G \cong H$ to mention that there is an isomorphism between two graphs $G$ and $H$. Also, if $G \cong H$, then we say $G$ and $H$ are isomorphic. A homomorphism from a graph $G$ to a graph $H$ is a mapping $f: V(G) \longrightarrow V(H)$ which preserves the adjacency, i.e., if $x y \in E(G)$, then $f(x) f(y) \in E(H)$. For brevity, we use $G \longrightarrow H$ to denote that there is a homomorphism from $G$ to $H$. If we have both $G \longrightarrow H$ and $H \longrightarrow G$, then we say $G$ and $H$ are homomorphically equivalent and show this by $G \longleftrightarrow H$. Note that $\chi(G)$ is the minimum integer $k$ for which there is a homomorphism from $G$ to the complete graph $K_{k}$. For a subgraph $H$ of $G$, the subgraph $G \backslash H$ is obtained from $G$ by removing the edge set of $H$. Also, $G-H$ is obtained from $G$ by removing the vertices of $H$ with their incident edges.

\section{$2.1 \quad$ Altermatic Number}

The sequence $x_{1}, x_{2}, \ldots, x_{m} \in\{-1,+1\}$ is said to be an alternating sequence, if any two consecutive terms are different. For any $X=\left(x_{1}, x_{2}, \ldots, x_{n}\right) \in\{-1,0,+1\}^{n} \backslash$ $\{(0,0, \ldots, 0)\}$, the alternation number of $X$, alt $(X)$, is the length of a longest al- 
ternating subsequence of nonzero terms of $\left(x_{1}, x_{1}, \ldots, x_{n}\right)$. Note that we consider just nonzero entries to determine the alternation number of $X$. However, for the simplicity of notations, we define alt $((0,0, \ldots, 0))=0$. Let $V=\left\{v_{1}, v_{2}, \ldots, v_{n}\right\}$ be a set of size $n$ and $L_{V}$ be the set of all linear orderings of $V$, i.e., $L_{V}=$ $\left\{v_{i_{1}}<v_{i_{2}}<\cdots<v_{i_{n}}:\left(i_{1}, i_{2}, \ldots, i_{n}\right) \in S_{n}\right\}$. For any linear ordering $\sigma: v_{i_{1}}<v_{i_{2}}<$ $\cdots<v_{i_{n}} \in L_{V}$ and $1 \leq j \leq n$, define $\sigma(j)=v_{i_{j}}$. We sometimes represent a linear ordering of $V$ by a permutation, i.e., $\sigma=\left(v_{i_{1}}, v_{i_{2}}, \ldots, v_{i_{n}}\right)$, and we use interchangeably these two kinds of representations of any linear ordering. Moreover, for any $X=\left(x_{1}, \ldots, x_{n}\right) \in\{-1,0,+1\}^{n}$, define $X_{\sigma}^{+}=\left\{\sigma(j): x_{j}=+1\right\}=\left\{v_{i_{j}}: x_{j}=+1\right\}$ and $X_{\sigma}^{-}=\left\{\sigma(k): x_{k}=-1\right\}=\left\{v_{i_{k}}: x_{k}=-1\right\}$.

For any hypergraph $\mathcal{H}=(V, E)$ and $\sigma \in L_{V}$, where $|V|=n$. Define $\operatorname{alt}_{\sigma}(\mathcal{H})$ (resp. $\left.\operatorname{salt}_{\sigma}(\mathcal{H})\right)$ to be the largest integer $k$ such that there exists an $X \in\{-1,0,+1\}^{n}$ with $\operatorname{alt}(X)=k$ and that none (resp. at most one) of $X_{\sigma}^{+}$and $X_{\sigma}^{-}$contains any (resp. some) hyperedge of $\mathcal{H}$. Note that if any singleton is a hyperedge of $\mathcal{H}$, then $\operatorname{alt}(\mathcal{H})=0$. Also, $\operatorname{alt}_{\sigma}(\mathcal{H}) \leq \operatorname{salt}_{\sigma}(\mathcal{H})$ and equality can hold. Now, set $\operatorname{alt}(\mathcal{H})=\min \left\{\operatorname{alt}_{\sigma}(\mathcal{H}): \sigma \in L_{V}\right\}$ and $\operatorname{salt}(\mathcal{H})=\min \left\{\operatorname{salt}_{\sigma}(\mathcal{H}): \sigma \in L_{V}\right\}$. Define the altermatic number and the strong altermatic number of a graph $G$, respectively, as follows

$$
\zeta(G)=\max _{\mathcal{H}}\{|V(\mathcal{H})|-\operatorname{alt}(\mathcal{H}): \operatorname{KG}(\mathcal{H}) \longleftrightarrow G\}
$$

and

$$
\zeta_{s}(G)=\max _{\mathcal{H}}\{|V(\mathcal{H})|+1-\operatorname{salt}(\mathcal{H}): \operatorname{KG}(\mathcal{H}) \longleftrightarrow G\} .
$$

It was proved in $[1,2]$ that both altermatic number and strong altermatic number provide tight lower bounds for the chromatic number of graphs.

Theorem A. [1] For any graph $G$, we have

$$
\chi(G) \geq \max \left\{\zeta(G), \zeta_{s}(G)\right\} .
$$

\subsection{Alternating Turán Number}

Let $G$ be a graph and $\mathcal{F}$ be a family of graphs. A subgraph of $G$ is called an $\mathcal{F}$ subgraph, if there is an isomorphism between this subgraph and a member of $\mathcal{F}$. The general Kneser graph $\operatorname{KG}(G, \mathcal{F})$ has all $\mathcal{F}$-subgraphs of $G$ as vertex set and two vertices are adjacent if the corresponding $\mathcal{F}$-subgraphs are edge-disjoint. A graph $G$ is said to be $\mathcal{F}$-free, if it has no subgraph isomorphic to a member of $\mathcal{F}$. For a graph $G$, define $\operatorname{ex}(G, \mathcal{F})$, the generalized Turán number of the family $\mathcal{F}$ in the graph $G$, to be the maximum number of edges of an $\mathcal{F}$-free spanning subgraph of $G$. A spanning subgraph of $G$ is called $\mathcal{F}$-extremal if it has $\operatorname{ex}(G, \mathcal{F})$-edges and it is $\mathcal{F}$-free. We denote the family of all $\mathcal{F}$-extremal subgraphs of $G$ with $\operatorname{EX}(G, \mathcal{F})$. It is usually a hard problem to determine the exact value of $\operatorname{ex}(G, \mathcal{F})$. The concept of Turán number was generalized in [2] in order to find the chromatic number of some families of general Kneser graphs as follows. Let $G$ be a graph with $E(G)=\left\{e_{1}, e_{2}, \ldots, e_{m}\right\}$. For any ordering $\sigma=\left(e_{i_{1}}, e_{i_{2}}, \ldots, e_{i_{m}}\right)$ of edges of $G$, a 2-coloring of a subset of edges of $G$ (with 2 colors red and blue) is said to be alternating (respect to the ordering $\sigma$ ), if any two consecutive colored edges (with respect to the ordering $\sigma$ ) receive different colors. Note that we may assign no color to some edges of $G$. In other 
words, in view of the ordering $\sigma$, we assign two colors red and blue alternatively to a subset of edges of $G$. We use the notation $\operatorname{ex}_{\text {alt }}(G, \mathcal{F}, \sigma)(\operatorname{resp}$. ex salt $(G, \mathcal{F}, \sigma))$ to denote the maximum number of edges of a spanning subgraph $H$ of $G$ such that the edges of $H$ can be colored alternatively (with respect to the ordering $\sigma$ ) by 2-colors so that each (resp. at least one of) color class is $\mathcal{F}$-free. Now, we are in a position to define the alternating Turán number $\operatorname{ex}_{a l t}(G, \mathcal{F})$ and the strong alternating Turán number $\operatorname{ex}_{\text {salt }}(G, \mathcal{F})$ as follows

$$
\operatorname{ex}_{\text {alt }}(G, \mathcal{F})=\min \left\{\operatorname{ex}_{\text {alt }}(G, \mathcal{F}, \sigma) ; \sigma \in L_{E(G)}\right\}
$$

and

$$
\operatorname{ex}_{\text {salt }}(G, \mathcal{F})=\min \left\{\operatorname{ex}_{\text {salt }}(G, \mathcal{F}, \sigma) ; \sigma \in L_{E(G)}\right\} .
$$

For a graph $G$, let $F$ be a member of $\operatorname{EX}(G, \mathcal{F})$ and $\sigma$ be an arbitrary ordering of $E(G)$. Now, if we color the edges of $F$ alternatively with two colors with respect to the ordering $\sigma$, one can see that any color class has no member of $\mathcal{F}$; and therefore, $\operatorname{ex}(G, \mathcal{F}) \leq \operatorname{ex}_{a l t}(G, \mathcal{F}, \sigma)$. Also, it is clear that if we assign colors to more than $2 \operatorname{ex}(G, \mathcal{F})$ edges, then a color class has at least more than $\operatorname{ex}(G, \mathcal{F})$ edges. It implies $\operatorname{ex}_{\text {alt }}(G, \mathcal{F}, \sigma) \leq 2 \operatorname{ex}(G, \mathcal{F})$. Consequently,

$$
\operatorname{ex}(G, \mathcal{F}) \leq \operatorname{ex}_{\text {alt }}(G, \mathcal{F}) \leq 2 \operatorname{ex}(G, \mathcal{F}) .
$$

Next lemma was proved in [2]. For the convenince of the reader, we repeat its proof, thus making our exposition self-contained.

Lemma A. [2] For any graph $G$ and family $\mathcal{F}$ of graphs,

$$
\begin{aligned}
|E(G)|-\operatorname{ex}_{\text {alt }}(G, \mathcal{F}) & \leq \chi(\operatorname{KG}(G, \mathcal{F})) \leq|E(G)|-\operatorname{ex}(G, \mathcal{F}), \\
|E(G)|+1-\operatorname{ex}_{\text {salt }}(G, \mathcal{F}) & \leq \chi(\operatorname{KG}(G, \mathcal{F})) \leq|E(G)|-\operatorname{ex}(G, \mathcal{F}) .
\end{aligned}
$$

Proof. Let $K \in E X(G, \mathcal{F})$. One can check that any vertex of $\operatorname{KG}(G, \mathcal{F})$ contains at least an element of $E(G) \backslash E(K)$. This implies $\chi(\operatorname{KG}(G, \mathcal{F})) \leq|E(G)|-\operatorname{ex}(G, \mathcal{F})$. On the other hand, consider the hypergraph $H$ whose vertex set is $E(G)$ and hyperedge set consists of all subgraphs of $G$ isomorphic to some member of $\mathcal{F}$. Note that $\mathrm{KG}(H)$ is isomorphic to $\operatorname{KG}(G, \mathcal{F})$. One can check that alt $(H)=\operatorname{ex}_{\text {alt }}(G, \mathcal{F})$ and $\operatorname{salt}(H)=\operatorname{ex}_{\text {salt }}(G, \mathcal{F})$. Now, in view of Theorem A, the assertion holds.

The previous lemma enables us to find the chromatic number of some families of graphs. If we present an appropriate ordering $\sigma$ of the edges of $G$ such that $\operatorname{ex}_{\text {alt }}(G, \mathcal{F})=\operatorname{ex}(G, \mathcal{F})$ or $\operatorname{ex}_{\text {salt }}(G, \mathcal{F})-1=\operatorname{ex}(G, \mathcal{F})$, then one can conclude that

$$
\chi(\operatorname{KG}(G, \mathcal{F}))=|E(G)|-\operatorname{ex}(G, \mathcal{F}) .
$$

By this observation, the chromatic number of several families of graphs was determined in [2].

Hereafter, for a given 2-coloring of a subset of edges of $G$, a spanning subgraph of $G$ whose edge set consists of all edges such that red (resp. blue) color has been assigned to them, is termed the red subgraph $G^{R}$ (resp. blue subgraph $G^{B}$ ). Furthermore, by abuse of language, any edge of $G^{R}$ (resp. $G^{B}$ ) is termed a red edge (resp. blue edge). 


\section{Matching Graphs}

In this section, we investigate the chromatic number of graphs via their altermatic number. First, in Subsection 3.1, we study the chromatic number of the matching graph $\operatorname{KG}\left(G, r K_{2}\right)$ when $G$ is a sparse graph. In contrast, in Subsection 3.2, we determine the chromatic number of the matching graph $\mathrm{KG}\left(G, r K_{2}\right)$ provided that $G$ is a large dense graph.

\subsection{A Generalization of Schrijver's Theorem}

Matching graphs can be considered as a generalization of Kneser, Schrijver, and permutation graphs. In fact, one can check that $\operatorname{KG}\left(n K_{2}, r K_{2}\right), \operatorname{KG}\left(C_{n}, r K_{2}\right)$, and $\mathrm{KG}\left(K_{m, n}, r K_{2}\right)$, are isomorphic to Kneser, Schrijver, and permutation graphs, respectively. Hence, as a generalization of Lovász's Theorem [12] and Schrijver's Theorem [13], it would be of interest to determine the chromatic number of matching graph $\operatorname{KG}\left(G, r K_{2}\right)$. It seems that for any graph $G$, we usually have $\chi\left(\operatorname{KG}\left(G, r K_{2}\right)\right)=$ $|E(G)|-\operatorname{ex}\left(G, r K_{2}\right)$, but the assertion is not true when $G$ is not a connected graph. For instance, note that $\chi\left(\mathrm{KG}\left(n K_{2}, r K_{2}\right)\right)=\left|E\left(n K_{2}\right)\right|-2 \operatorname{ex}\left(n K_{2}, r K_{2}\right)$. In this section, we introduce some sufficient conditions such that the equality $\chi\left(\operatorname{KG}\left(G, r K_{2}\right)\right)=$ $|E(G)|-\operatorname{ex}\left(G, r K_{2}\right)$ holds.

A famous generalization of Tutte's Theorem by Berge in 1985, says that the largest number of vertices saturated by a matching in $G$ is $\min _{S \subseteq V(G)}\{|V(G)|-o(G-$ $S)+|S|\}$, where $o(G-S)$ is the number of odd components in $G-S$. For a bipartite graph, we define its odd girth to be infinite.

Theorem 1. Let $r \geq 2$ be an integer and $G$ be a connected graph with odd girth at least $g$, vertex set $V(G)=\left\{v_{1}, v_{2}, \ldots, v_{n}\right\}$, and degree sequence $\operatorname{deg}_{G}\left(v_{1}\right) \geq$ $\operatorname{deg}_{G}\left(v_{2}\right) \geq \cdots \geq \operatorname{deg}_{G}\left(v_{n}\right)$. Moreover, suppose that $r \leq \max \left\{\frac{g}{2}, \frac{\operatorname{deg}_{G}\left(v_{r-1}\right)+1}{4}\right\}$ and $\left\{v_{1}, \ldots, v_{r-1}\right\}$ forms an independent set. If $\operatorname{deg}_{G}\left(v_{r-1}\right)$ is an even integer or $\operatorname{deg}_{G}\left(v_{r-1}\right)>\operatorname{deg}_{G}\left(v_{r}\right)$, then $\chi\left(\operatorname{KG}\left(G, r K_{2}\right)\right)=|E(G)|-\sum_{i=1}^{r-1} \operatorname{deg}_{G}\left(v_{i}\right)$.

Proof. Consider the subgraph of $G$ containing of all edges incident to some $v_{i}$, for $1 \leq i \leq r-1$. This subgraph does not have any $r$-matching; consequently, we have $\chi\left(\operatorname{KG}\left(G, r K_{2}\right)\right) \leq|E(G)|-\sum_{i=1}^{r-1} \operatorname{deg}_{G}\left(v_{i}\right)$. Hence, it is sufficient to show that $G$ satisfies $\chi\left(\operatorname{KG}\left(G, r K_{2}\right)\right) \geq|E(G)|-\sum_{i=1}^{r-1} \operatorname{deg}_{G}\left(v_{i}\right)$. Set $s$ to be the number of $v_{i}$ 's such that $\operatorname{deg}_{G}\left(v_{i}\right)$ is an odd integer, where $1 \leq i \leq r-1$. If $G$ is an even graph, set $H=G$; otherwise, add a new vertex $w$ and join it to any odd vertex of $G$ to obtain the graph $H$. Now, $H$ has an Eulerian tour $e_{1}^{\prime}, e_{2}^{\prime}, \ldots, e_{m}^{\prime}$, where if $G$ is not an even graph, we start the Eulerian tour with $w$; otherwise, it starts with $v_{n}$. Consider the ordering $\left(e_{1}^{\prime}, e_{2}^{\prime}, \ldots, e_{m}^{\prime}\right)$ and remove all new edges incident with $w$ from this ordering to obtain the ordering $\sigma$ for the edge set of $G$. In other words, if we traverse the edge $e_{i}$ before than the edge $e_{j}$ in the Eulerian tour, then in the 
ordering $\sigma$ we have $e_{i}<e_{j}$. Now, consider an alternating coloring (with colors blue and red) of edges of $G$ with respect to the ordering $\sigma$ of length $t$, where if $s \neq 0$, then $t=1+\sum_{i=1}^{r-1} \operatorname{deg}_{G}\left(v_{i}\right)$; otherwise, $t=2+\sum_{i=1}^{r-1} \operatorname{deg}_{G}\left(v_{i}\right)$. Recall that $G^{R}$ and $G^{B}$ are the spanning subgraphs of $G$ whose edge sets consist of all red edges and blue edges, respectively. We show that if $s=0$, then each of $G^{R}$ and $G^{B}$ has an $r$-matching; and consequently, $\operatorname{ex}_{\text {salt }}\left(G, r K_{2}, \sigma\right) \leq 1+\sum_{i=1}^{r-1} \operatorname{deg}_{G}\left(v_{i}\right)$. Also, we show that, if $s \neq 0$, then $G^{R}$ or $G^{B}$ has an $r$-matching; and consequently, $\operatorname{ex}_{a l t}\left(G, r K_{2}, \sigma\right) \leq \sum_{i=1}^{r-1} \operatorname{deg}_{G}\left(v_{i}\right)$. In view of Lemma A, these, these imply $\chi\left(\operatorname{KG}\left(G, r K_{2}\right)\right)=|E(G)|-\sum_{i=1}^{r-1} \operatorname{deg}_{G}\left(v_{i}\right)$. In view of the ordering $\sigma$, one can see that for any vertex $x$ of $G$ (except at most the first vertex of the Eulerian tour), color red (resp. blue) can be assigned to at most half of edges adjacent to $x$, i.e., $\left\lceil\frac{\operatorname{deg}_{G}(x)}{2}\right\rceil$. For the first vertex, if $t$ is an even integer, then any color can be assigned to at most half of edges incident to it. This amount can be increased by at most one when $t$ is an odd integer. Now, the proof falls into two parts.

a) First, we show that if $r \leq \frac{g}{2}$, then the assertion holds. In fact, if $s \neq 0$, then we determine the chromatic number by evaluating the altermatic chromatic number. Otherwise, we show that the strong altermatic chromatic number is equal to chromatic number. For $j \in\{R, B\}$, if $G^{j}$ does not have any $r$ matching, then in view of the Tutte-Berge Formula, there exists an $S^{j} \subseteq$ $V\left(G^{j}\right)=V(G)$ such that $\left|V\left(G^{j}\right)\right|-o\left(G^{j}-S^{j}\right)+\left|S^{j}\right| \leq 2 r-2$. Suppose that $O_{1}^{j}, O_{2}^{j}, \ldots, O_{t_{j}}^{j}$ are the components of $G^{j}-S^{j}$. One can check that for each $1 \leq i \leq t_{j},\left|V\left(O_{i}^{j}\right)\right| \leq 2 r-\left|S^{j}\right|-1 \leq g-1$. Therefore, every component $O_{i}^{j}$ does not have any odd cycle and so it would be a bipartite graph. Assume that $O_{i}^{j}=O_{i}^{j}\left(X_{i}^{j}, Y_{i}^{j}\right)$ such that $\left|X_{i}^{j}\right| \leq\left|Y_{i}^{j}\right|\left(X_{i}^{j}\right.$ may be an empty set). Set $X^{j}=\cup_{i=1}^{t_{j}} X_{i}^{j}$. Note that

$$
\left|X^{j}\right|=\sum_{i=1}^{t_{j}}\left\lfloor\frac{\left|V\left(O_{i}^{j}\right)\right|}{2}\right\rfloor \leq \frac{\left|V\left(G^{j}\right)\right|-\left|S^{j}\right|}{2}-\frac{\left|V\left(G^{j}\right)\right|+\left|S^{j}\right|-2 r+2}{2}=r-\left|S^{j}\right|-1 .
$$

Therefore, for $s=0$ and any $j \in\{R, B\}$, if $G^{j}$ does not have any $r$-matching, then

$$
\begin{aligned}
1+\sum_{i=1}^{r-1} \frac{\operatorname{deg}_{G}\left(v_{i}\right)}{2} & =\left|E\left(G^{j}\right)\right| \\
& \leq \sum_{x \in S^{j}}\left\lceil\frac{\operatorname{deg}_{G}(x)}{2}\right\rceil+\sum_{x \in X^{j}}\left\lceil\frac{\operatorname{deg}_{G}(x)}{2}\right\rceil \\
& \leq \sum_{i=1}^{r-1} \frac{\operatorname{deg}_{G}\left(v_{i}\right)}{2}
\end{aligned}
$$


which is impossible. This means that $\operatorname{ex}_{\text {salt }}\left(G, r K_{2}, \sigma\right) \leq 1+\sum_{i=1}^{r-1} \operatorname{deg}_{G}\left(v_{i}\right)$. Accordingly, $\chi\left(\operatorname{KG}\left(G, r K_{2}\right)\right)=|E(G)|-\sum_{i=1}^{r-1} \operatorname{deg}_{G}\left(v_{i}\right)$. Now, assume that $s \neq 0$. Also, suppose that neither $G^{R}$ nor $G^{B}$ has any r-matching. In view of the assumption, $\operatorname{deg}_{G}\left(v_{r-1}\right)$ is an even integer or $\operatorname{deg}_{G}\left(v_{r-1}\right)>\operatorname{deg}_{G}\left(v_{r}\right)$. Hence,

$$
\begin{aligned}
1+\sum_{i=1}^{r-1} \operatorname{deg}_{G}\left(v_{i}\right) & =\left|E\left(G^{R}\right)\right|+\left|E\left(G^{B}\right)\right| \\
& \leq \sum_{j} \sum_{x \in S^{j}} \operatorname{deg}_{G^{j}}(x)+\sum_{j} \sum_{x \in X^{j}} \operatorname{deg}_{G^{j}}(x) \\
& \leq \sum_{i=1}^{r-1} \operatorname{deg}_{G}\left(v_{i}\right)
\end{aligned}
$$

which is a contradiction. This means that $\operatorname{ex}_{\text {alt }}\left(G, r K_{2}, \sigma\right) \leq \sum_{i=1}^{r-1} \operatorname{deg}_{G}\left(v_{i}\right)$. Accordingly, $\chi\left(\operatorname{KG}\left(G, r K_{2}\right)\right)=|E(G)|-\sum_{i=1}^{r-1} \operatorname{deg}_{G}\left(v_{i}\right)$.

b) Now, we show that if $r \leq \frac{\operatorname{deg}_{G}\left(v_{r-1}\right)+1}{4}$, then the assertion holds. For $j \in$ $\{R, B\}$, if $G^{j}$ does not have any $r$-matching, in view of the Tutte-Berge Formula, there exists an $S^{j} \subseteq V\left(G^{j}\right)$ such that $\left|V\left(G^{j}\right)\right|-o\left(G^{j}-S^{j}\right)+\left|S^{j}\right| \leq$ $2 r-2$. Assume that $O_{1}^{j}, O_{2}^{j}, \ldots, O_{t_{j}}^{j}$ are all components of $G^{j}-S^{j}$, where $t_{j} \geq o\left(G^{j}-S^{j}\right)$. We consider two different cases $s=0$ and $s>0$. First assume that $s=0$ and so $t=2+\sum_{i=1}^{r-1} \operatorname{deg}_{G}\left(v_{i}\right)$. Now, we show that each of $G^{R}$ and $G^{B}$ has a matching of size $r$. Assume that $G^{R}$ (resp. $G^{B}$ ) does not have any matching of size $r$. Therefore,

$$
\begin{aligned}
\left|E\left(G^{R}\right)\right| & \leq \sum_{x \in S^{R}} \operatorname{deg}_{G^{R}}(x)+\sum_{i=1}^{t_{R}}\left(\begin{array}{c}
\left|V\left(O_{i}^{R}\right)\right| \\
2
\end{array}\right) \\
& \leq \sum_{x \in S^{R}} \operatorname{deg}_{G^{R}}(x)+\left(\begin{array}{c}
\sum_{i=1}^{t_{R}}\left|V\left(O_{i}^{R}\right)\right|-\left(t_{R}-1\right) \\
2
\end{array}\right) \\
& \leq \sum_{x \in S^{R}} \operatorname{deg}_{G^{R}}(x)+\left(\begin{array}{c}
2 r-2\left|S^{R}\right|-1 \\
2
\end{array}\right) \\
& \leq \frac{1}{2} \sum_{i=1}^{r-1} \operatorname{deg}_{G}\left(v_{i}\right)
\end{aligned}
$$

which is impossible. Now, assume that $s \neq 0$. We show that $G^{R}$ or $G^{B}$ has a matching of size $r$. On the contrary, suppose that both $G^{R}$ and $G^{B}$ does not 
have any matching of size $r$. First, suppose that $\left|S^{R}\right| \neq r-1$ or $\left|S^{B}\right| \neq r-1$. Note that $1+\sum_{i=1}^{r-1} \operatorname{deg}_{G}\left(v_{i}\right)=t=\left|E\left(G^{R}\right)\right|+\left|E\left(G^{B}\right)\right|$. On the other hand,

$$
\begin{aligned}
t & \leq \sum_{j} \sum_{x \in S^{j}} \operatorname{deg}_{G^{j}}(x)+\sum_{j} \sum_{i=1}^{t_{j}}\left(\begin{array}{c}
\left|V\left(O_{i}^{j}\right)\right| \\
2
\end{array}\right) \\
& \leq \sum_{j} \sum_{x \in S^{j}} \operatorname{deg}_{G^{j}}(x)+\sum_{j}\left(\sum_{i=1}^{t_{j}}\left|V\left(O_{i}^{j}\right)\right|-\left(t_{j}-1\right)\right. \\
& \leq \sum_{j} \sum_{i=1}^{\left|S^{j}\right|} \frac{\operatorname{deg}_{G}\left(v_{i}\right)}{2}+\min \left\{\frac{r-1}{2}, \frac{\left|S^{R}\right|+\left|S^{B}\right|}{2}\right\}+\sum_{j}\left(\begin{array}{c}
2 r-2\left|S^{j}\right|-1 \\
2
\end{array}\right) \\
& \leq \sum_{j} \sum_{i=1}^{\left|S^{j}\right|} \frac{\operatorname{deg}_{G}\left(v_{i}\right)}{2}+\sum_{j}\left(r-\left|S^{j}\right|-1\right) \frac{\operatorname{deg}_{G}\left(v_{r-1}\right)}{2} \\
& \leq \sum_{i=1}^{r-1} \operatorname{deg}_{G}\left(v_{i}\right)
\end{aligned}
$$

which is impossible. If $\left|S^{R}\right|=\left|S^{B}\right|=r-1$, then each connected component of $G^{j}-S^{j}$ is a single vertex. Hence,

$$
1+\sum_{i=1}^{r-1} \operatorname{deg}_{G}\left(v_{i}\right) \leq\left|E\left(G^{R}\right)\right|+\left|E\left(G^{B}\right)\right| \leq \sum_{j} \sum_{x \in S^{j}} \operatorname{deg}_{G^{j}}(x) \leq \sum_{i=1}^{r-1} \operatorname{deg}_{G}\left(v_{i}\right),
$$

a contradiction. Consequently, $\operatorname{ex}_{a l t}\left(G, r K_{2}, \sigma\right) \leq \sum_{i=1}^{r-1} \operatorname{deg}_{G}\left(v_{i}\right)$; and accordingly, $\chi\left(\mathrm{KG}\left(G, r K_{2}\right)\right)=|E(G)|-\sum_{i=1}^{r-1} \operatorname{deg}_{G}\left(v_{i}\right)$.

Note that $\operatorname{KG}\left(C_{n}, r K_{2}\right) \cong \mathrm{SG}(n, r)$. Hence, the aforementioned theorem can be considered as a generalization of Schrijver's Theorem [13].

Theorem B. [13] For any positive integers $n$ and $r$, where $n \geq 2 r$, we have $\chi(\mathrm{SG}(n, r))=n-2 r+2$.

Corollary 1. Let $G$ be a connected non-bipartite $k$-regular graph with odd girth at least $g$, where $k$ is an even integer. For any positive integer $r \leq \frac{g}{2}$, we have $\chi\left(\operatorname{KG}\left(G, r K_{2}\right)\right)=|E(G)|-k(r-1)$.

Proof. Let $C$ be a minimal odd cycle in $G$. Note that $C$ is an induced subgraph of $G$ and $|V(C)| \geq g$. Consequently, it contains an independent set of size $\left\lfloor\frac{g}{2}\right\rfloor$. Therefore, in view of Theorem 1, the proof is completed. 


\subsection{Matching-Dense Graphs}

In this subsection, we determine the chromatic number of matching-dense graphs.

Let $G$ be a graph with $V(G)=\left\{u_{1}, \ldots, u_{n}\right\}$. The graph $G$ is termed $(r, c)$ locally Eulerian, if there are edge-disjoint nontrivial Eulerian connected subgraphs $H_{1}, \ldots, H_{n}$ of $G$ such that for any $1 \leq i \leq n$, we have $u_{i} \in H_{i}$ and that for any $u \in V\left(H_{i}\right)$, where $u \neq u_{i}$, we have $\operatorname{deg}_{H_{i}}\left(u_{i}\right) \geq(r-1) \operatorname{deg}_{H_{i}}(u)+c$.

Lemma 1. Let $r \geq 2$ and $s$ be nonnegative integers. Also, let $G$ be a graph with $n$ vertices and $\delta(G)>\left(\begin{array}{c}r+2 \\ 2\end{array}\right)+(r-2) s$. If there exists an $(r+s, c)$-locally Eulerian graph $H$ such that $G$ is a subgraph of $H,|V(H)|=|V(G)|+s$, and $c \geq\left(\begin{array}{c}r-1 \\ 2\end{array}\right)+(s+3)(r-1)$, then $\chi\left(\mathrm{KG}\left(G, r K_{2}\right)\right)=\zeta\left(\operatorname{KG}\left(G, r K_{2}\right)\right)=|E(G)|-\operatorname{ex}\left(G, r K_{2}\right)$.

Proof. In view of Lemma $A$, it is sufficient to show that there exists an ordering $\sigma$ of the edges of $G$ such that $\operatorname{ex}_{\text {alt }}\left(G, r K_{2}, \sigma\right)=\operatorname{ex}\left(G, r K_{2}\right)$.

Let $V(G)=\left\{u_{1}, \ldots, u_{n}\right\}$ and $V(H)=\left\{u_{1}, \ldots, u_{n+s}\right\}$. In view of definition of $(r, c)$-locally Eulerian graph, there are pairwise edge-disjoint nontrivial Eulerian subgraphs $H_{1}, \ldots, H_{n+s}$ of $H$ such that for any $1 \leq i \leq n+s$, we have $u_{i} \in H_{i}$ and that for any $u \in V\left(H_{i}\right)$, where $u \neq u_{i}$, we have $\operatorname{deg}_{H_{i}}\left(u_{i}\right) \geq(r+s-1) \operatorname{deg}_{H_{i}}(u)+$ $\left(\begin{array}{c}r-1 \\ 2\end{array}\right)+(s+3)(r-1)$.

To find the ordering $\sigma$, add a new vertex $x$ and join it to all vertices of $H$ by edges with multiplicity two to obtain the graph $H^{\prime}$. Precisely, for any $1 \leq i \leq n+s$, join $x$ and $u_{i}$ with two distinct edges $f_{i}$ and $f_{i}^{\prime}$. Now, if $H^{\prime}$ has no odd vertices, then set $\bar{H}=H^{\prime}$; otherwise, add a new vertex $z$ to $H^{\prime}$ and join it to all odd vertices of $H^{\prime}$ to obtain the graph $\bar{H}$. The graph $\bar{H}$ is an even graph; and therefore, it has an Eulerian tour.

Also, note that the graph $K=\bar{H}-x$ is an even graph; and accordingly, any connected component of $K^{\prime}=K \backslash\left(\bigcup_{i=1}^{n+s} H_{i}\right)$ is also an Eulerian subgraph. Without loss of generality, assume that $K_{1}, \ldots, K_{l}$ are the connected components of $K^{\prime}$. Construct an Eulerian tour for $\bar{H}$ as follows. At $i^{\text {th }}$ step, where $1 \leq i \leq n+s$, start from the vertex $x$ and traverse the edge $f_{i}$. Consider an arbitrary Eulerian tour of $H_{i}$ starting at $u_{i}$ and traverse it. Next, if there exists a $1 \leq j \leq l$ such that $u_{i} \in K_{j}$ and the edge set of $K_{j}$ is still untraversed, then consider an Eulerian tour for $K_{j}$ starting at $u_{i}$ and traverse it. Next, traverse the edge $f_{i}^{\prime}$ and if $i<n+s$, then start $(i+1)^{\text {th }}$ step. Construct an ordering $\sigma$ for the edge set of the graph $G$ such that the ordering of edges in $E(G)$ are corresponding to their ordering in the aforementioned Eulerian tour, i.e., if we traverse the edge $e_{i} \in E(G)$ before the edge $e_{j} \in E(G)$ in the Eulerian tour, then in the ordering $\sigma$ we have $e_{i}<e_{j}$.

Now, we claim that $\operatorname{ex}_{a l t}\left(G, r K_{2}, \sigma\right)=\operatorname{ex}\left(G, r K_{2}\right)$. Note that for any $r-1$ vertices $\left\{u_{i_{1}}, \ldots, u_{i_{r-1}}\right\} \subseteq V(G)$, we have

$$
\operatorname{ex}\left(G, r K_{2}\right) \geq \sum_{j=1}^{r-1} \operatorname{deg}_{G}\left(u_{i_{j}}\right)-\left(\begin{array}{c}
r-1 \\
2
\end{array}\right) .
$$

Consider an alternating 2-coloring of the edges of $G$ with respect to the ordering $\sigma$ of length $\operatorname{ex}\left(G, r K_{2}\right)+1$, i.e., we assigned $\operatorname{ex}\left(G, r K_{2}\right)+1$ blue and red colors 
alternatively to the edges of $G$ with respect to the ordering $\sigma$. For a contradiction, suppose that both red spanning subgraph $G^{R}$ and blue spanning subgraph $G^{B}$ are $r K_{2}$-free subgraphs. In view of the Berge-Tutte Formula, there are two sets $T^{R}$ and $T^{B}$ such that $\left|V\left(G^{R}\right)\right|-o\left(G^{R}-T^{R}\right)+\left|T^{R}\right| \leq 2 r-2$ and $\left|V\left(G^{B}\right)\right|-o\left(G^{B}-\right.$ $\left.T^{B}\right)+\left|T^{B}\right| \leq 2 r-2$. In view of these inequalities, one can see that $\left|T^{R}\right| \leq r-1$ and $\left|T^{B}\right| \leq r-1$. Moreover, the number of edges of $G^{R}$ not incident to some vertex of $T^{R}\left(\left|E\left(G^{R}-T^{R}\right)\right|\right)$ is at most $\left(\begin{array}{c}2 r-2\left|T^{R}\right|-1 \\ 2\end{array}\right)$. To see this, assume that $O_{1}^{R}, O_{2}^{R}, \ldots, O_{t_{R}}^{R}$ are all connected components of $G^{R}-T^{R}$, where $t_{R} \geq o\left(G^{R}-T^{R}\right) \geq$ $\left|V\left(G^{R}\right)\right|+\left|T^{R}\right|-2 r+2$. We have

$$
\begin{aligned}
\left|E\left(G^{R}-T^{R}\right)\right| \leq \sum_{i=1}^{t_{R}}\left(\begin{array}{c}
\left|V\left(O_{i}^{R}\right)\right| \\
2
\end{array}\right) & \leq\left(\begin{array}{c}
\sum_{i=1}^{t_{R}}\left|V\left(O_{i}^{R}\right)\right|-\left(t_{R}-1\right) \\
2
\end{array}\right) \\
& \leq \quad\left(\begin{array}{c}
2 r-2\left|T^{R}\right|-1 \\
2
\end{array}\right) .
\end{aligned}
$$

Similarly, we show $\left|E\left(G^{B}-T^{B}\right)\right| \leq\left(\begin{array}{c}2 r-2\left|T^{B}\right|-1 \\ 2\end{array}\right)$. Also, in view of the ordering $\sigma$, any vertex $u$ of $G$ is incident to at most $\frac{1}{2}\left(\operatorname{deg}_{G}(u)+s+3\right)$ edges of $G^{R}$ (resp. $\left.G^{B}\right)$. First, we show that if $\left|T^{R}\right| \leq r-2$ or $\left|T^{B}\right| \leq r-2$, then the assertion holds. If $\left|T^{R}\right| \leq r-2$, then

$$
\begin{aligned}
\left|E\left(G^{R}\right)\right| & \leq\left(\begin{array}{c}
2 r-2\left|T^{R}\right|-1 \\
2
\end{array}\right)+\sum_{u \in T^{R}} \frac{1}{2}\left(\operatorname{deg}_{G}(u)+s+3\right) \\
& \leq\left(\begin{array}{c}
2 r-2\left|T^{R}\right|-1 \\
2
\end{array}\right)+\frac{\operatorname{ex}\left(G, r K_{2}\right)}{2}-\frac{r-1-\left|T^{R}\right|}{2} \delta(G)+\frac{1}{2}\left(\begin{array}{c}
r-1 \\
2
\end{array}\right)+\frac{(s+3)\left|T^{R}\right|}{2} \\
& <\frac{\operatorname{ex}\left(G, r K_{2}\right)}{2} .
\end{aligned}
$$

Since $\left|E\left(G^{B}\right)\right| \leq\left|E\left(G^{R}\right)\right|+1$, we have $\operatorname{ex}_{\text {alt }}\left(G, r K_{2}, \sigma\right)=\left|E\left(G^{R}\right)\right|+\left|E\left(G^{B}\right)\right| \leq$ $\operatorname{ex}\left(G, r K_{2}\right)$ which is impossible. Similarly, if $\left|T^{B}\right| \leq r-2$, then the assertion holds.

If $\left|T^{R}\right|=\left|T^{B}\right|=r-1$, in view of the Berge-Tutte Formula, one can conclude that all connected components of $G^{R}-T^{R}$ and $G^{B}-T^{B}$ are isolated vertices. This means that $T^{R}$ and $T^{B}$ are vertex covers of $G^{R}$ and $G^{B}$, respectively. Now, we show that $T^{R}=T^{B}$. One the contrary, suppose that $T^{R} \neq T^{B}$. Without loss of generality, let $\left|E\left(G^{R}\right)\right| \geq\left|E\left(G^{B}\right)\right|$ and choose a vertex $u_{i} \in T^{R} \backslash T^{B}$.

Set $L^{B}=\left|E\left(H_{i}^{B}\right)\right|$, i.e., the number of blue edges of $H_{i}$. Since $T^{B}$ is a vertex cover of $G^{B}$ and all edges of $H_{i}$ appear consecutively in the ordering $\sigma$ (according to an Eulerian tour of $H_{i}$ ), one can check that at least $2 L^{B}$ edges of $H_{i}$ are incident to the vertices of $T^{B}$. It implies that there is a vertex $z \in V\left(H_{i}\right) \backslash\left\{u_{i}\right\}$ with degree at least $\frac{2 L^{B}}{r-1}$. However, by the assumption that for any $u \in V\left(H_{i}\right) \backslash\left\{u_{i}\right\}$,

$$
\operatorname{deg}_{H_{i}}\left(u_{i}\right) \geq(r+s-1) \operatorname{deg}_{H_{i}}(u)+\left(\begin{array}{c}
r-1 \\
2
\end{array}\right)+(s+3)(r-1),
$$

we have

$$
\operatorname{deg}_{H_{i}}\left(u_{i}\right) \geq(r+s-1)\left\lceil\frac{2 L^{B}}{r-1}\right\rceil+\left(\begin{array}{c}
r-1 \\
2
\end{array}\right)+(s+3)(r-1) .
$$


Thus, we have

$$
L^{B} \leq \frac{1}{2}\left(\operatorname{deg}_{H_{i}}\left(u_{i}\right)-\left(\begin{array}{c}
r-1 \\
2
\end{array}\right)-(s+3)(r-1)\right) .
$$

Note that red color can be assigned to at most one edge between any two consecutive edge of $G$ in the ordering $\sigma$. Define $l^{R}$ to be the number of red edges incident to $u_{i}$ in the subgraph $H_{i}$. One can see that $l^{R} \leq L^{B}+1$. If there exists a $1 \leq j \leq l$ such that $u_{i} \in K_{j}$ and $u_{r} \notin K_{j}$ for any $r<i$, then set $H_{i}^{\prime}=H_{i} \cup K_{j}$; otherwise, define $H_{i}^{\prime}=H_{i}$. Now, we show that the number of red edges incident to $u_{i}$ in the graph $H_{i}^{\prime}$ is at most $L^{B}+1+\frac{1}{2} \operatorname{deg}_{H_{i}^{\prime}}\left(u_{i}\right)-\frac{1}{2} \operatorname{deg}_{H_{i}}\left(u_{i}\right)$. If $H_{i}^{\prime}=H_{i}$, then there is nothing to prove. Otherwise, one can check that red color can be assigned to at most $\frac{1}{2} \operatorname{deg}_{H_{i}^{\prime}}\left(u_{i}\right)-\frac{1}{2} \operatorname{deg}_{H_{i}}\left(u_{i}\right)+1$ edges incident to $u_{i}$ in the subgraph $H_{i}^{\prime} \backslash H_{i}$. Moreover, if $l^{R}=L^{B}+1$, then one can see that there are two red edges $e$ and $e^{\prime}$ in $H_{i}$ such that $e$ (resp. $e^{\prime}$ ) appears before (resp. after) any edge of $H_{i}^{B}$ in the ordering $\sigma$. Consequently, in this case, red color can be assigned to at most $\frac{1}{2} \operatorname{deg}_{H_{i}^{\prime}}\left(u_{i}\right)-\frac{1}{2} \operatorname{deg}_{H_{i}}\left(u_{i}\right)$ edges incident to $u_{i}$ in the subgraph $H_{i}^{\prime} \backslash H_{i}$. Thus, the number of red edges incident to $u_{i}$ in the graph $H_{i}^{\prime}$ is at most $L^{B}+1+\frac{1}{2} \operatorname{deg}_{H_{i}^{\prime}}\left(u_{i}\right)-\frac{1}{2} \operatorname{deg}_{H_{i}}\left(u_{i}\right)$. Moreover, in view of the ordering of the Eulerian tour of $\bar{H}$, one can conclude that red color can be assigned to at most $\frac{1}{2}\left(\operatorname{deg}_{G}\left(u_{i}\right)+s+1-\operatorname{deg}_{H_{i}^{\prime}}\left(u_{i}\right)\right)$ edges incident to $u_{i}$ in the subgraph $G \backslash H_{i}^{\prime}$. Therefore, the number of red edges incident to $u_{i}$ in the graph $G$ is at most

$$
\begin{aligned}
& L^{B}+1+\frac{1}{2} \operatorname{deg}_{H_{i}^{\prime}}\left(u_{i}\right)-\frac{1}{2} \operatorname{deg}_{H_{i}}\left(u_{i}\right)+\frac{1}{2}\left(\operatorname{deg}_{G}\left(u_{i}\right)+s+1-\operatorname{deg}_{H_{i}^{\prime}}\left(u_{i}\right)\right) \leq \\
& \frac{1}{2}\left(\operatorname{deg}_{G}\left(u_{i}\right)-(s+3)(r-2)-\left(\begin{array}{c}
r-1 \\
2
\end{array}\right)\right)
\end{aligned}
$$

Consequently,

$$
\begin{aligned}
\left|E\left(G^{R}\right)\right| & \leq \frac{1}{2}\left(\operatorname{deg}_{G}\left(u_{i}\right)-(s+3)(r-2)-\left(\begin{array}{c}
r-1 \\
2
\end{array}\right)\right)+\sum_{u \in T^{R} \backslash\left\{u_{i}\right\}} \frac{1}{2}\left(\operatorname{deg}_{G}(u)+s+3\right) \\
& \leq-\frac{1}{2}\left(\begin{array}{c}
r-1 \\
2
\end{array}\right)+\sum_{u \in T^{R}} \frac{1}{2} \operatorname{deg}_{G}(u) \\
& \leq \frac{1}{2} \operatorname{ex}\left(G, r K_{2}\right)
\end{aligned}
$$

and it is a contradiction. Hence, $T^{R}=T^{B}$. Therefore, the number of blue and red edges of $G$, i.e., $\left|E\left(G^{R}\right)\right|+\left|E\left(G^{B}\right)\right|$, is at most the number of edges incident with vertices in $T^{R}$ or $T^{B}$ which is at $\operatorname{most} \operatorname{ex}\left(G, r K_{2}\right)$ and this is impossible. Accordingly, $\operatorname{ex}_{a l t}\left(G, r K_{2}, \sigma\right)=\operatorname{ex}\left(G, r K_{2}\right)$.

Let $G$ be a graph. A $G$-decomposition of a graph $H$ is a set $\left\{G_{1}, \ldots, G_{t}\right\}$ of pairwise edge-disjoint subgraphs of $H$ such that for each $1 \leq i \leq t$, the graph $G_{i}$ is isomorphic to $G$; and moreover, the edge sets of $G_{i}$ 's partition the edge set of $H$. A $G$-decomposition of $H$ is called a monogamous $G$-decomposition, if any distinct pair of vertices of $H$ appear in at most one copy of $G$ in the decomposition. Note that if a graph $H$ has a decomposition into the complete graph $K_{t}$, then it is a monogamous $K_{t}$-decomposition. 
Theorem C. [11] Let $m$ and $n$ be positive even integers. The complete bipartite graph $K_{m, n}$ has a monogamous $C_{4}$-decomposition if and only if $(m, n)=(2,2)$ or $6 \leq n \leq m \leq 2 n-2$.

Lemma 2. Let $r, t$ and $t^{\prime}$ be positive integers, where $11 \leq t \leq t^{\prime} \leq 2 t-2$. If $c$ is a nonnegative integer and $t \geq 8 r+4 c+2$, then the complete bipartite graph $K_{t, t^{\prime}}$ is $(r, c)$-locally Eulerian.

Proof. Assume that $t=2 p+q$ and $t^{\prime}=2 p^{\prime}+q^{\prime}$, where $0 \leq q \leq 1$ and $0 \leq q^{\prime} \leq 1$. Extend the complete bipartite graph $G=K_{t, t^{\prime}}$ to the complete bipartite graph $H=K_{T, T^{\prime}}$, where $T=t+q$ and $T^{\prime}=t^{\prime}+q^{\prime}$. In view of Theorem C, consider a monogamous $C_{4}$-decomposition of $H$. Call any $C_{4}$ of this decomposition a block, if it is entirely in $G$. Construct a bipartite graph with the vertex set $(U, V)$ such that $U$ consists of $\left\lfloor\frac{t-3}{8}\right\rfloor$ copies of each vertex of $K_{t, t^{\prime}}$ and $V$ consists of all blocks. Join a vertex of $U$ to a vertex of $V$, if the corresponding vertex of $K_{t, t^{\prime}}$ is contained in the corresponding block. One can check that the degree of each vertex in the part $U$ is at least $\left\lceil\frac{t-3}{2}\right\rceil$ and the degree of any vertex in the part $V$ is $4\left\lfloor\frac{t-3}{8}\right\rfloor$. In view of Hall's Theorem, one can see that this bipartite graph has a matching which saturates all vertices of $U$. For any vertex $v \in K_{t, t^{\prime}}$, consider $\left\lceil\frac{t-3}{8}\right\rceil$ blocks assigned to $v$ through the perfect matching and set $H_{v}$ to be a subgraph of $K_{t, t^{\prime}}$ formed by the union of these blocks. One can see that $\operatorname{deg}_{H_{v}}(v)=2\left\lfloor\frac{t-3}{8}\right\rfloor$, while the degree of any other vertices of $H_{v}$ is 2 . In view of $H_{v}$ 's, one can see that $K_{t, t^{\prime}}$ is an $(r, c)$-locally Eulerian graph.

For a family $\mathcal{F}$ of graphs, we say a graph $G$ has an $\mathcal{F}$-factor if there are vertexdisjoint subgraphs $H_{1}, H_{2}, \ldots, H_{t}$ of $G$ such that each $H_{i}$ is a member of $\mathcal{F}$ and $\bigcup_{i=1}^{t} V\left(H_{i}\right)=V(G)$. Note that if a graph $G$ has an $\mathcal{F}$-factor, where each member of $\mathcal{F}$ is an $(r, c)$-locally Eulerian graph, then $G$ is also $(r, c)$-locally Eulerian. In view of the aforementioned lemma, if a graph $G$ has a $K_{t, t^{\prime}}$ factor, then one can conclude that $G$ is $(r, c)$-locally Eulerian. Now, we introduce some sufficient condition for a graph to have a $K_{t, t^{\prime}}$ factor.

Graph expansion was studied extensively in the literature. Let $0<\nu \leq \tau<1$ and assume that $G$ is a graph with $n$ vertices. For $S \subseteq V(G)$, the $\nu$-robust neighborhood of $S, R N_{\nu, G}(S)$, is the set of all vertices $v \in V(G)$ such that $\left|N_{G}(v) \cap S\right| \geq \nu n$. A graph $G$ is called robust $(\nu, \tau)$-expander, if for any $S \subseteq V(G)$ with $\tau n \leq|S| \leq(1-\tau) n$ we have $\left|R N_{\nu, G}(S)\right| \geq|S|+\nu n$. Throughout this section, we write $0<a \ll b \ll c$ to mean that we can choose the constants $a, b$, and $c$ from right to left. More precisely, there are two increasing functions $f$ and $g$ such that, given $c$, whenever we choose some $b \leq g(c)$ and $a \leq f(b)$, for more about robust $(\nu, \tau)$-expander see [7]. A graph $G$ with $n$ vertices has bandwidth at most $b$ if there exists a bijective assignment $l: V(G) \longrightarrow[n]$ such that for every edge $u v \in E(G)$, we have $|l(u)-l(v)| \leq b$.

Theorem D. [7] Let $\nu, \tau$, and $\eta$ be real numbers, where $0<\nu \leq \tau \ll \eta<1$, and $\Delta$ be a positive integer. There exist constants $\beta>0$ and $n_{0}$ such that the following holds. Let $H$ be a bipartite graph on $n \geq n_{0}$ vertices with $\Delta(H) \leq \Delta$ and bandwidth at most $\beta n$. If $G$ is a robust $(\nu, \tau)$-expander with $n$ vertices and $\delta(G) \geq \eta n$, then $G$ contains a copy of $H$. 
Theorem 2. Let $\nu, \tau$ and $\eta$ be real numbers, where $0<\nu \leq \tau \ll \eta<1$. If $n$ is sufficiently large, then for any robust $(\nu, \tau)$-expander graph $G$ with $n$ vertices and $\delta(G) \geq \eta n, \chi\left(\operatorname{KG}\left(G, r K_{2}\right)\right)=\zeta\left(\operatorname{KG}\left(G, r K_{2}\right)\right)=|E(G)|-\operatorname{ex}\left(G, r K_{2}\right)$.

Proof. In view of Lemma 1, it is sufficient to show that the graph $G$ is an $(r, c)$ locally Eulerian graph, where $c=\left(\begin{array}{c}r-1 \\ 2\end{array}\right)+3(r-1)$. Set $t=2 r^{2}+14 r-6$ and let $k$ and $t^{\prime}$ be integers such that $n=2 t k+t^{\prime}$ and $2 t \leq t^{\prime} \leq 4 t-1$. Now, set $H$ to be a bipartite graph on $n$ vertices with $1+\frac{n-t^{\prime}}{2 t}$ connected components such that one component is isomorphic to $K_{\left\lceil\frac{t^{\prime}}{2}\right\rceil,\left\lfloor\frac{t^{\prime}}{2}\right\rfloor}$ and any other component is isomorphic to $K_{t, t}$. In view of Theorem D, if $n$ is sufficiently large, then $H$ is a spanning subgraph of $G$. By Lemma 2, one can see that $K_{\left\lceil\frac{t^{\prime}}{2}\right\rceil,\left\lfloor\frac{t^{\prime}}{2}\right\rfloor}$ and $K_{t, t}$ are both $(r, c)$-locally Eulerian graph; consequently, by Lemma 1, the theorem follows.

The following lemma is an immediate consequence of Lemma 13 from [10].

Lemma B. [10] For positive constants $\tau \ll \eta<1$, there exists an integer $n_{0}$ such that if $G$ is a graph with $n \geq n_{0}$ vertices and the degree sequence $d_{1} \leq d_{2} \leq \cdots \leq d_{n}$ such that for any $i<\frac{n}{2}, d_{i} \geq i+\eta n$ or $d_{n-i-\lfloor\eta n\rfloor} \geq n-i$, then $\delta(G) \geq \eta n$ and $G$ is a robust $\left(\tau^{2}, \tau\right)$-expander.

In view of the previous lemma and Theorem 2, we have the next corollary.

Corollary 2. For a positive constant $\gamma$, there is an integer $n_{0}$ such that for any $n \geq n_{0}$ we have the followings. If $G$ is a connected graph with $n$ vertices and the degree sequence $d_{1} \leq d_{2} \leq \cdots \leq d_{n}$ such that that for each $i<\frac{n}{2}$, we have $d_{i} \geq i+\gamma n$ or $d_{n-i-\lfloor\gamma n\rfloor} \geq n-i$, then $\chi\left(\mathrm{KG}\left(G, r K_{2}\right)\right)=|E(G)|-\operatorname{ex}\left(G, r K_{2}\right)$.

For a graph property $\mathcal{P}$, we say $G(n, p)$ possesses $\mathcal{P}$ asymptotically almost sure, or a.a.s. for brevity, if the probability that $G \in G(n, p)$ possesses the property $\mathcal{P}$ tends to 1 as $n$ tends to infinity. For constants $0<\nu \ll \tau \ll p<1$, a.a.s. any graph $G$ in $G(n, p)$ is a robust $(\nu, \tau)$-expander graph with minimum degree at least $\frac{p n}{2}$ and maximum degree at most $2 n p$. This observation and Theorem 2 imply that a.a.s. for any graph $G$ in $G(n, p)$ we have $\chi\left(\operatorname{KG}\left(G, r K_{2}\right)\right)=|E(G)|-\operatorname{ex}\left(G, r K_{2}\right)$. Moreover, Huang, Lee, and Sudakov [6] proved a more general theorem. Here, we state it in a special case.

Theorem E. [6] For positive integers $r, \Delta$ and reals $0<p \leq 1$ and $\gamma>0$, there exists a constant $\beta>0$ such that a.a.s., any spanning subgraph $G^{\prime}$ of any $G \in G(n, p)$ with minimum degree $\delta\left(G^{\prime}\right) \geq p\left(\frac{1}{2}+\gamma\right) n$ contains every $n$-vertex bipartite graph $H$ which has the maximum degree at most $\Delta$ and bandwidth at most $\beta n$.

In view of the proof of Theorem 2 and the previous theorem, we have the following result.

Corollary 3. If $0<p \leq 1$ and $\gamma>0$, then a.a.s. for any spanning subgraph $G^{\prime}$ of any $G \in G(n, p)$ with minimum degree at least $p\left(\frac{1}{2}+\gamma\right) n$ we have $\chi\left(\operatorname{KG}\left(G^{\prime}, r K_{2}\right)\right)=$ $\left|E\left(G^{\prime}\right)\right|-\operatorname{ex}\left(G^{\prime}, r K_{2}\right)$. 
Let $H$ be a graph with $h$ vertices and $\chi(H)=l$. Set $\operatorname{cr}(H)$ to be the size of the smallest color class over all proper $l$-colorings of $H$. The critical chromatic number, $\chi_{\mathrm{cr}}(H)$, is defined as $(l-1) \frac{h}{h-\mathrm{cr}(H)}$. One can check that $\chi(H)-1<$ $\chi_{\mathrm{cr}}(H) \leq \chi(H)$ and equality holds in the upper bound if and only if in any $l$ coloring of $H$, all color classes have the same size. Assume that $H$ has $k$ connected components $C_{1}, C_{2}, \ldots, C_{k}$. Define $\operatorname{hcf}_{c}(H)$ to be the highest common factor of integers $\left|C_{1}\right|,\left|C_{2}\right|, \ldots,\left|C_{k}\right|$. Let $f$ be an $l$-coloring of $H$ such that $x_{1} \leq x_{2} \leq \cdots \leq x_{l}$ are the size of coloring classes in $f$. Set $D(f)=\left\{x_{i+1}-x_{i} \mid 1 \leq i \leq l-1\right\}$ and $D(H)=\bigcup D(f)$ where the union ranges over all $l$-colorings $f$ of $H$. Now, define $\operatorname{hcf}_{\chi}(H)$ to be the highest common factor of the members of $D(H)$. If $D(H)=\{0\}$, then we define $\operatorname{hcf}_{\chi}(H)=\infty$. We say

$$
H \text { is in class } 1 \text { if } \begin{cases}\operatorname{hcf}_{\chi}(H)=1 & \text { when } \chi(H) \neq 2, \\ \operatorname{hcf}_{\chi}(H) \leq 2 \text { and } \operatorname{hcf}_{c}(H)=1 & \text { when } \chi(H)=2,\end{cases}
$$

otherwise, $H$ is in Class 2.

Theorem F. [9] For every graph $H$ on $h$ vertices, there are integers $c$ and $m_{0}$ such that for all integers $m \geq m_{0}$, if $G$ is a graph on $n=m h$ vertices, then the following holds. If

$$
\delta(G) \geq \begin{cases}\left(1-\frac{1}{\chi_{\mathrm{cr}}(H)}\right) n+c & H \text { is in Class 1, } \\ \left(1-\frac{1}{\chi(H)}\right) n+c & H \text { is in Class 2, }\end{cases}
$$

then $G$ has an $H$-factor.

Theorem 3. For any integer $r \geq 2$, there are constants $\alpha, \beta$, and $n_{0}$ such that for any graph $G$ with $n$ vertices, if $\delta(G) \geq\left(\frac{1}{2}-\alpha\right) n+\beta$ and $n \geq n_{0}$, then $\chi\left(\mathrm{KG}\left(G, r K_{2}\right)\right)=\zeta\left(\mathrm{KG}\left(G, r K_{2}\right)\right)=|E(G)|-\operatorname{ex}\left(G, r K_{2}\right)$.

Proof. Define $t=2 r^{2}+14 r-6$. Set $H$ to be a bipartite graph with two connected components $C_{1}$ and $C_{2}$ isomorphic to $K_{t, t}$ and $K_{t+1, t}$, respectively. One can check that $H$ is in Class 1 . Hence, by Theorem F, there are integers $c_{1}$ and $m_{1}$ such that if $\left|V\left(G^{\prime}\right)\right| \geq m_{1},|V(H)|$ divides $\left|V\left(G^{\prime}\right)\right|$, and $\delta\left(G^{\prime}\right) \geq\left(1-\frac{1}{\chi_{\mathrm{cr}}(H)}\right)\left|V\left(G^{\prime}\right)\right|+c_{1}$, then the graph $G^{\prime}$ has an $H$-factor. Let $T$ be an integer such that $4 t+1 \leq T<8 t+2$ and $4 t+1 \mid n-T$. It is known that if $n$ is sufficiently large and $\delta(G) \geq \eta n$, where $0<\eta<1$, then $G$ contains a copy of the complete bipartite graph $K_{\left\lceil\frac{T}{2}\right\rceil\left\lfloor\frac{T}{2}\right\rfloor}$. Note that $\frac{1}{\chi_{\mathrm{cr}}(H)}=\frac{1}{2}+\frac{1}{8 t+2}$. Set $\alpha=\frac{1}{8 t+2}$ and $\beta=c_{1}+8 t-1$. If $\delta(G) \geq\left(\frac{1}{2}-\alpha\right) n+\beta$ and $n$ is sufficiently large, then $G$ contains $K_{\left\lceil\frac{T}{2}\right\rceil,\left\lfloor\frac{T}{2}\right\rfloor}$ and also the graph $G \backslash K_{\left\lceil\frac{T}{2}\right\rceil,\left\lfloor\frac{T}{2}\right\rfloor}$ has an $H$-factor. Hence, $G$ can be decomposed into the complete bipartite graphs $K_{t, t}$, $K_{t+1, t}$, and $K_{\left\lceil\frac{T}{2}\right\rceil,\left\lfloor\frac{T}{2}\right\rfloor}$. In view of Lemma 2, these graphs are $(r, c)$-locally Eulerian graphs with $c=\left(\begin{array}{c}r-1 \\ 2\end{array}\right)+3(r-1)$. Therefore, $G$ is an $(r, c)$-locally Eulerian graph; and consequently, by Lemma 1, the assertion holds.

\subsection{Permutation Graphs}

Let $m, n, r$ be positive integers, where $r \leq m, n$. For an $r$-subset $A \subseteq[m]$ and an injective map $f: A \longrightarrow[n]$, the ordered pair $(A, f)$ is said to be an $r$-partial 
permutation [4]. Let $S_{r}(m, n)$ denotes the set of all $r$-partial permutations. Two partial permutations $(A, f)$ and $(B, g)$ are said to be intersecting, if there exists an $x \in A \cap B$ such that $f(x)=g(x)$. Note that $S_{n}(n, n)$ is the set of all $n$ permutations. The permutation graph $S_{r}(m, n)$ has all $r$-partial permutations $(A, \sigma)$ as its vertex set and two $r$-partial permutations are adjacent if and only if they are not intersecting. Note that $S_{r}(m, n) \cong S_{r}(n, m)$; and therefore, for the simplicity, we assume that $m \geq n$ for all permutation graphs. One can see that the permutation graph $S_{r}(m, n)$ is isomorphic to $\mathrm{KG}\left(K_{m, n}, r K_{2}\right)$.

Next theorem gives a sufficient condition for a balanced bipartite graph to have a decomposition into complete bipartite subgraphs.

Theorem G. [14] For any integer $q \geq 2$, there exists a positive integer $m_{0}$ such that for all $m \geq m_{0}$, the following holds. If $G=(X, Y)$ is a balanced bipartite graph on $2 n=2 m q$ vertices, i.e., $|X|=|Y|=m q$, with

$$
\delta(G) \geq \begin{cases}\frac{n}{2}+q-1 & \text { if } m \text { is even } \\ \frac{n+3 q}{2}-2 & \text { if } m \text { is odd }\end{cases}
$$

then $G$ has a $K_{q, q^{-}}$factor.

Now, we investigate the chromatic number of general Kneser graph $\mathrm{KG}\left(G, r K_{2}\right)$ provided that $G=(X, Y)$ is a balanced $(|X|=|Y|)$ dense bipartite graph. In particular, we determine the chromatic number of any permutation graph provided that the number of its vertices is large enough. For more about permutation graphs, see $[3,5,8]$.

Theorem 4. For any positive integer $r$, there exist constants $q$ and $m$ such that for all $n \geq m$, the following holds. If $G$ is a graph on $2 n$ vertices which has a bipartite subgraph $H=(U, V)$ with $|U|=|V|=n$ and $\delta(H) \geq \frac{n}{2}+q$, then $\chi\left(\operatorname{KG}\left(G, r K_{2}\right)\right)=$ $\zeta\left(\mathrm{KG}\left(G, r K_{2}\right)\right)=|E(G)|-\operatorname{ex}\left(G, r K_{2}\right)$.

Proof. In view of Lemma 1, it is sufficient to show that the graph $H$ is an $(r, c)$ locally Eulerian graph, where $c=\left(\begin{array}{c}r-1 \\ 2\end{array}\right)+3(r-1)$. Set $t=2 r^{2}+14 r-6$. By Theorem $\mathrm{G}$, there are integers $q_{1}$ and $m_{1}$ such that if $n \geq m_{1}$ and $t \mid n$, then any balanced bipartite graph $H^{\prime}$ with $2 n$ vertices and $\delta\left(H^{\prime}\right) \geq \frac{n}{2}+q_{1}$ has a $K_{t, t}$-factor.

Let $t^{\prime}$ be an integer, where $t \leq t^{\prime}<2 t$ and $t \mid n-t^{\prime}$. It is known that if $n$ is sufficiently large and $\delta(H) \geq \eta n$, where $0<\eta<1$, then $H$ contains a copy of the complete bipartite graph $K_{t^{\prime}, t^{\prime}}$. Define $q=q_{1}+2 t-1$. Note that if $n$ is sufficiently large, then $H$ contains a copy of $K_{t^{\prime},{ }^{\prime} t}$ and also, in view of Theorem G, $H \backslash K_{t^{\prime}, t^{\prime}}$ has a $K_{t, t}$-factor. This implies that $H$ can be decomposed into complete bipartite graphs $K_{t^{\prime}, t^{\prime}}$ and $K_{t, t}$. In view of Lemma 2, these graphs are $(r, c)$-locally Eulerian graphs, where $c=\left(\begin{array}{c}r-1 \\ 2\end{array}\right)+3(r-1)$. Therefore, $H$ and $G$ are $(r, c)$-locally Eulerian graphs; and consequently, by Lemma 1, the assertion holds.

Corollary 4. Let $m, n, r$ be positive integers, where $m \geq n \geq r$. If $m$ is large enough, then

$$
\chi\left(\mathrm{KG}\left(K_{m, n}, r K_{2}\right)\right)=\zeta\left(\mathrm{KG}\left(K_{m, n}, r K_{2}\right)\right)=m(n-r+1) .
$$


Proof. In view of Hall's Theorem, any maximal $r K_{2}$-free subgraph of $K_{m, n}$ has $(r-1) m$ edges. Hence, in view of Lemma A, we have $\chi\left(\operatorname{KG}\left(K_{m, n}, r K_{2}\right)\right) \leq m(n-$ $r+1)$. In view of Lemma 4 , if $m$ is sufficiently large, then $\chi\left(\operatorname{KG}\left(K_{m, m}, r K_{2}\right)\right)=$ $m(m-r+1)$. Now, we show that for any positive integer $n<m$, if $m$ is sufficiently large, then $\chi\left(\operatorname{KG}\left(K_{m, n}, r K_{2}\right)\right)=m(n-r+1)$. To see this, on the contrary, suppose that $f: V\left(\mathrm{KG}\left(K_{m, n}, r K_{2}\right)\right) \longrightarrow\left\{1,2, \ldots, \chi\left(\mathrm{KG}\left(K_{m, n}, r K_{2}\right)\right)\right\}$ is a proper coloring of $\mathrm{KG}\left(K_{m, n}, r K_{2}\right)$, where $\chi\left(\mathrm{KG}\left(K_{m, n}, r K_{2}\right)\right)<m(n-r+1)$. Add $m-n$ new vertices to the small part of $K_{m, n}$ and join them to all vertices in the other part to construct $K_{m, m}$, and call the new edges $e_{1}, \ldots, e_{(m-n) m}$. Extend the coloring $f$ to a proper coloring $g$ for $\mathrm{KG}\left(K_{m, m}, r K_{2}\right)$ as follows. If a matching $M$ is a subset of $K_{m, n}$, then set $g(M)=f(M)$; otherwise, assume that $i$ is the smallest positive integer such that $e_{i} \in M$, in this case set $g(M)=i+\chi\left(\operatorname{KG}\left(K_{m, n}, r K_{2}\right)\right)$. This provides a proper coloring for $\mathrm{KG}\left(K_{m, m}, r K_{2}\right)$ with less than $m(m-r+1)$ colors which is a contradiction.

Let $s \geq t$ be positive integers and $G=G(X, Y)$ be a connected $(s, t)$-regular connected bipartite graph. Theorem 1 implies that if $s$ is an even integer, then for any $r \leq|X|$ we have $\chi\left(\operatorname{KG}\left(G, r K_{2}\right)\right)=s(|X|-r+1)$. This result shows that $\chi\left(\operatorname{KG}\left(K_{m, n}, r K_{2}\right)\right)=\chi\left(S_{r}(m, n)\right)=m(n-r+1)$ provided that $m$ is an even integer and $m \geq n \geq r$. However, if $m$ is a small odd value, then the chromatic number of the permutation graph $S_{r}(m, n)$ is unknown.

Corollary 5. Let $m, n, r$ be positive integers, where $m \geq n \geq r$. If $m$ is even, then

$$
\chi\left(\mathrm{KG}\left(K_{m, n}, r K_{2}\right)\right)=\zeta\left(\mathrm{KG}\left(K_{m, n}, r K_{2}\right)\right)=m(n-r+1) .
$$

The aforementioned results motivate us to consider the following conjecture.

Conjecture 1. For any connected graph $G$ and positive integer $r$, we have

$$
\chi\left(\mathrm{KG}\left(G, r K_{2}\right)\right)=|E(G)|-\operatorname{ex}\left(G, r K_{2}\right) .
$$

Acknowledgement: The authors would like to express their deepest gratitude to Professor Carsten Thomassen for his insightful comments. They also appreciate the detailed valuable comments of Dr. Saeed Shaebani. This paper was written while Hossein Hajiabolhassan was visiting School of Mathematics, Institute for Research in Fundamental Sciences (IPM). He acknowledges the support of IPM. Moreover, they would like to thank Skype for sponsoring their endless conversations in two countries.

\section{References}

[1] Meysam Alishahi and Hossein Hajiabolhassan. On the chromatic number of general kneser hypergraphs. Journal of Combinatorial Theory, Series B (2015), http://dx.doi.org/10.1016/j.jctb.2015.05.010.

[2] M. Alishahi and H. Hajiabolhassan. Chromatic number via Turán number. ArXiv e-prints, December 2013. 
[3] Peter J. Cameron and C. Y. Ku. Intersecting families of permutations. European J. Combin., 24(7):881-890, 2003.

[4] Xing Bo Geng, Jun Wang, and Hua Jun Zhang. Structure of independent sets in direct products of some vertex-transitive graphs. Acta Math. Sin. (Engl. Ser.), 28(4):697-706, 2012.

[5] Chris Godsil and Karen Meagher. A new proof of the Erdős-Ko-Rado theorem for intersecting families of permutations. European J. Combin., 30(2):404-414, 2009 .

[6] Hao Huang, Choongbum Lee, and Benny Sudakov. Bandwidth theorem for random graphs. J. Combin. Theory Ser. B, 102(1):14-37, 2012.

[7] Fiachra Knox and Andrew Treglown. Embedding spanning bipartite graphs of small bandwidth. Combin. Probab. Comput., 22(1):71-96, 2013.

[8] C. Y. Ku and I. Leader. An Erdős-Ko-Rado theorem for partial permutations. Discrete Math., 306(1):74-86, 2006.

[9] Daniela Kühn and Deryk Osthus. The minimum degree threshold for perfect graph packings. Combinatorica, 29(1):65-107, 2009.

[10] Daniela Kühn, Deryk Osthus, and Andrew Treglown. Hamiltonian degree sequences in digraphs. J. Combin. Theory Ser. B, 100(4):367-380, 2010.

[11] C. C. Lindner and A. Rosa. Monogamous decompositions of complete bipartite graphs, symmetric H-squares, and self-orthogonal 1-factorizations. Australas. J. Combin., 20:251-256, 1999.

[12] L. Lovász. Kneser's conjecture, chromatic number, and homotopy. J. Combin. Theory Ser. A, 25(3):319-324, 1978.

[13] A. Schrijver. Vertex-critical subgraphs of Kneser graphs. Nieuw Arch. Wisk. (3), 26(3):454-461, 1978.

[14] Yi Zhao. Bipartite graph tiling. SIAM J. Discrete Math., 23(2):888-900, 2009. 